\title{
Ultrasound: The Requisites: Third Edition Barbara S. and Hertzberg William D. Middleton
}

Ultrasound: The Requisites is a stellar reference textbook co-authored by Barbara S. Hertzberg and William D. Middleton. Both authors have a well-established reputation in the field of ultrasound and are recognized internationally for their competence and knowledge. Maintaining their clear and compelling style of writing, they have succeeded in simplifying complex issues and understanding of crucial concepts. In addition, the extent of work put into the third edition to augment the content with many new illustrations and images is highly commendable.

The book is divided into two parts - General and Vascular \& Obstetrics and Gynecology - with content spread over 24 chapters. A new chapter addressing ultrasound evaluation of chromosomal abnormalities has been added to the Obstetrics and Gynecology section. The physics chapter has been completely rewritten, taking into account the changing needs of students by focusing on clinically relevant material. Significant updates have also been made to many chapters to reflect advancements in the field of ultrasound.
Another major enhancement is the e-book version that is included with the print edition. A symbol alerts the reader when a relevant video is available, and the reader can then take advantage of excellent digital resources that complement the textbook's images. The videos are very easy to navigate to and are guaranteed to greatly enhance understanding of the material.

At the core of this book lies a commitment to address all aspects of ultrasound pertinent to the needs of radiology residents and to serve as a reference book for practicing radiologists in the community. As with prior editions, the third edition continues to be an excellent resource for all who seek a comprehensive textbook of ultrasound.

\section{Nirvikar Dahiya}

Department of Radiology, Mayo Clinic, Phoenix, AZ, USA email: dahiya.nirvikar@mayo.edu 